\title{
Redes de turismo de base comunitária: reflexões no contexto latino-americano
}

\section{Community-based tourism networks: reflections in the Latin American context}

\author{
Edilaine Albertino Moraes, Marta de Azevedo Irving, Joana da Silva Castro Santos, \\ Hugo Quintanilha Silva Santos, Maycon Correia Pinto
}

\begin{abstract}
RESUMO
O desenvolvimento de iniciativas designadas como turismo de base comunitária (TBC) vem ocorrendo, de maneira progressiva, na América Latina. Essa proposta tem como premissa fundamental a base endógena em planejamento e desenvolvimento do turismo que tem sido interpretada como uma oportunidade para a melhoria de qualidade de vida por inúmeros grupos sociais, como pescadores artesanais, etnias indígenas, agricultores familiares, populações extrativistas, camponeses, entre outros, que vivem em situação de vulnerabilidade social e ambiental e à margem de processos dominantes de projetos turísticos. Outro aspecto importante associado às iniciativas de TBC são as estratégias políticas de grupos organizados e de movimentos sociais para a garantia e a preservação de territórios por eles ocupados tradicionalmente, como acontece com os movimentos indígenas e do campo em diversos países da América Latina. Esses processos coletivos têm contribuído para que o TBC venha sendo organizado por meio de coletivos, redes e alianças locais, nacionais e latino-americanas. Um exemplo emblemático desse tipo de organização do TBC nessa região tem se configurado por meio da Rede de Turismo Comunitário da América Latina (REDTURS), que foi criada em 2001, com o objetivo de articular redes que vêm sendo construídas em diferentes escalas local e nacional, com a finalidade de diversificar as possibilidades de emprego e renda, de valorizar a cultura local e de fomentar o associativismo. Essa iniciativa vem influenciando outras práticas na região. Com base nesses antecedentes, o presente artigo tem o objetivo de refletir sobre como se configura o movimento de TBC em rede na América Latina, com ênfase no caso da REDTURS. Para tal, a metodologia adotada se baseou em pesquisa bibliográfica e documental para orientar a fundamentação conceitual sobre o tema. Para a interpretação do caso da REDTURS, buscou-se também a análise de documentação técnica vinculada a essa experiência no período de 2001 a 2008. Os dados obtidos na pesquisa indicam inúmeras fragilidades e potencialidades no estabelecimento de relações e alianças formais e informais, baseadas em princípios de solidariedade, para a promoção de intercâmbio e compartilhamento de informação, conhecimento e recursos no processo de construção coletiva de redes no campo do turismo de base comunitária.
\end{abstract}

PALAVRAS-CHAVE: Redes; Turismo de Base Comunitária; Alianças; Intercâmbio; América Latina. 


\section{ABSTRACT}

The development of initiatives known as community-based tourism (CBT) has been taking place gradually, in Latin America. This proposal has as its fundamental premise, the endogenous basis on planning and development of tourism which has been interpreted as an opportunity to improve quality of life for countless social groups such as artisanal fishermen, indigenous groups, family farmers, extractive population, peasants, among others living in situations of social and environmental vulnerability and in the margins of the dominant processes of tourism projects. Another important aspect associated with CBT initiatives are political strategies of organized groups and social movements to guarantee the preservation of the territories traditionally occupied by them, as it happens with indigenous and rural movements in several Latin American countries. These collective processes have been contributing to the CBT organization through local, national and Latin American collective, local networks and alliances. An emblematic example of this type of CBT organization in this region has been set up through the Community Tourism Network of Latin America (REDTURS), which was established in 2001 with the objective of coordinating networks that have been built in different local and national levels, with the purpose of diversifying sources of employment and income, valuing local culture and promoting partnerships. This initiative has been influencing other practices in the region. Based on this background, this article aims to reflect on how to configure the movement of the CBT network in Latin America, with emphasis in the case of REDTURS. For this, the methodology adopted was based on bibliographical and documentary research to guide the conceptual foundation on the subject. For the interpretation of the case of REDTURS, it was also sought the analysis of technical documentation linked to this experience in the period from 2001 to 2008. The data obtained in the survey indicate numerous weaknesses and capabilities of the relations and formal and informal alliances, based on principles of solidarity to promote exchange and sharing of information, knowledge and resources in the process of collective construction of networks in the communitybased tourism field.

KEYWORDS: Networks, Community Based Tourism; Alliances; Exchange; Latin America.

\section{Introdução}

Iniciativas designadas como turismo de base comunitária, turismo comunitário ou turismo rural comunitário (TBC) vêm se construindo, em várias regiões do mundo e também na América Latina, como forma de resistência e como alternativa ao modelo convencional, na tentativa de se delinearem novas possibilidades para o desenvolvimento turístico, ou seja, na busca por um "outro turismo possível" (NEUHAUS; SILVA, 2006). Essa proposta, que tem como premissa fundamental a base endógena em planejamento e desenvolvimento do turismo na América Latina, tem sido interpretada como uma oportunidade para a melhoria de qualidade de vida para inúmeros grupos sociais, como pescadores artesanais, etnias indígenas, agricultores familiares, populações extrativistas, entre outros, que vivem em situação de vulnerabilidade social e ambiental e à margem de processos dominantes de projetos turísticos (MENDONÇA; MORAES, 2012). 
Em muitos casos, o TBC tem representado estratégias políticas de grupos organizados e de movimentos sociais para a garantia de manutenção e de preservação de territórios por eles ocupados tradicionalmente (LIMA; CORIOLANO, 2003). Esses processos têm contribuído para que o TBC venha sendo organizado por meio de coletivos, redes e alianças locais, nacionais e latino-americanas.

Um exemplo emblemático desse tipo de organização do TBC nessa região tem se configurado por meio da Rede de Turismo Comunitário da América Latina (REDTURS), que foi criada em 2001, com o objetivo de articular redes que vêm sendo construídas em diferentes escalas local e nacional, com a finalidade de diversificar as possibilidades de emprego e renda, de valorizar a cultura local e de fomentar o associativismo. Essa iniciativa vem influenciando outras práticas de turismo de base comunitária na região, tendo em vista os laços históricos e culturais dos países desde o México até o Polo Sul.

Com base nesses antecedentes, o presente artigo tem o objetivo de refletir sobre como se configura o movimento de TBC em rede na América Latina, com ênfase no caso da REDTURS. Para tal, a metodologia adotada se baseou em pesquisa bibliográfica e documental para orientar a fundamentação conceitual sobre o tema. Para a interpretação do caso da REDTURS, buscou-se também a análise de documentação técnica vinculada a essa experiência no período de 2001 a 2008. Os dados obtidos na pesquisa indicam inúmeras fragilidades $e$ potencialidades no estabelecimento de relações e alianças formais e informais, baseadas em princípios de solidariedade, para a promoção de intercâmbio e compartilhamento de informação, de conhecimento e de recursos no processo de construção coletiva de redes no campo do turismo de base comunitária.

Sob esta abordagem, o artigo está estruturado em duas seções principais. A primeira aborda a fundamentação teórica norteadora para esta reflexão, envolvendo as principais questões conceituais sobre turismo de base comunitária, para orientar a análise do caso da REDTURS, a partir de seus princípios e relevância no contexto latino-americano. Em seguida, busca-se caracterizar a REDTURS, considerando-se o seu contexto de criação e a sua perspectiva atual de atuação em rede, por meio das iniciativas participantes articuladas no nível local e nacional.

\section{Turismo de base comunitária na América Latina: Questões conceituais orientadoras para a atuação em rede}

As primeiras experiências de TBC na América Latina surgiram, na década de 1980, por iniciativa de populações rurais de origem indígena da região dos Andes e da América Central, sendo essas associadas ao reconhecimento da importância de elementos socioculturais singulares e à grande beleza natural paisagística da região (MALDONADO, 2009).

Nos dias atuais, as iniciativas de TBC ocorrem em quase todos os países latino-americanos, envolvendo várias áreas de elevada biodiversidade, mais de 300 destinos e cerca de 500 comunidades rurais e 
indígenas, com destaque para as experiências desenvolvidas no Equador, no Brasil, na Nicarágua e no Peru (MALDONADO, 2010). Esses grupos sociais, em conjunto com instituições de apoio e profissionais do setor, vêm discutindo e promovendo, desde 2001, inúmeras ações em torno do TBC, por meio da Rede de Turismo Comunitário da América Latina (REDTURS), impulsionada pela Organização Internacional do Trabalho (OIT).

Segundo Maldonado (2009), a origem do TBC na América Latina se deve a diversos fatores de ordem econômica, social, cultural e política, os quais se relacionam, sobretudo, à pressão do mercado turístico internacional; à busca pela superação de uma situação de pobreza crônica; ao papel relevante das micro e pequenas empresas na sustentação do desenvolvimento local. Ademais, as estratégias políticas dos movimentos dos povos indígenas e das populações rurais para a preservação de seus territórios ancestrais, que resistem, frequentemente, aos projetos governamentais de desenvolvimento, às ações colonizadoras de ocupação de "novas terras", aos empreendimentos de extração de recursos florestais, minerais e petrolíferos e às empresas de exploração agrícola e pecuária.

Orientadas pelo marco dos direitos coletivos dos povos indígenas e tribais consagrados pela Convenção 169 da OIT (1989), as experiências de TBC se baseiam em princípios derivados do reconhecimento e da proteção da visão de mundo (cosmovisão) própria desses povos sobre os valores e práticas sociais, culturais, religiosas e espirituais na relação natureza e cultura. Para eles, a terra e os seres humanos são indissociáveis e estão ligados por um mesmo destino para garantir a harmonia do mundo que deve ser constantemente recriada, transcendendo o tempo e os indivíduos. Esses princípios e valores estão expressos na Declaración de San José sobre Turismo Rural Comunitario, documento assinado por representantes comunitários da Bolívia, Brasil, Costa Rica, Equador, Guatemala e Peru, durante o II Encuentro Latino-Americano de Turismo Comunitário, realizado na Costa Rica, em 2003, pela REDTURS. Nesse documento, a concepção de TBC se baseia nos valores de solidariedade, cooperação, respeito pela vida e conservação e uso sustentável dos recursos naturais (MALDONADO, 2009) ${ }^{1}$.

Por essa perspectiva, o TBC se fundamenta "na propriedade e na autogestão sustentável dos recursos patrimoniais comunitários, de acordo com as práticas de cooperação e equidade no trabalho e na distribuição dos benefícios gerados pela prestação dos serviços turísticos" (MALDONADO, 2009, p.30).

O diferencial da experiência de TBC seria a sua dimensão humana e cultural, que busca incentivar o diálogo entre iguais e os encontros interculturais entre visitantes e visitados, na perspectiva de conhecer e de aprender com seus respectivos modos de vida (MALDONADO, 2009). Nesse caso, busca-se valorizar as manifestações das identidades culturais em articulação com a vivência dos atrativos naturais, históricos e recreativos.

No que tange ao processo de planejamento e gestão dessas iniciativas, investigações empíricas têm apontado que um diferencial positivo em experiências turísticas de base comunitária tem sido, em geral, o alto 
nível de participação e de articulação comunitária, motivado pela luta e pela resistência aos problemas e riscos sociais, políticos, territoriais, ambientais, econômicos e culturais no contexto em que estes estão inseridos (MENDONÇA; IRVING, 2004). Esse processo tende, assim, a exigir um nível de participação que possa transformar o indivíduo e o grupo social em protagonistas de sua própria história.

Porém, mesmo reconhecendo o elevado grau de participação das populações locais no desenvolvimento do TBC na América Latina, Maldonado (2009) salienta que existem ainda várias deficiências e riscos que limitam a potencialidade das iniciativas em curso. Nesse caso, é preciso reconhecer a limitação dessas populações com relação à formação profissional, ao acesso aos serviços públicos, à infraestrutura básica e à capacidade de gestão de negócios, que limita a afirmação dessas iniciativas no mercado turístico global. Embora a criação de "empresas em rede", na perspectiva horizontalizada de configuração (agrupamentos, parcerias ou clusters), tenha sido difundida para apoiar a promoção comercial do TBC no âmbito da REDTURS, parece haver um consenso de que apenas as populações envolvidas e as organizações de apoio não podem enfrentar, por si sós, os desafios para a consolidação dessas iniciativas latino-americanas.

O desenvolvimento do TBC na América Latina implica também a formulação de políticas públicas adequadas às reais demandas nesse campo. Nesse caso, Maldonado (2009) ressalta que Equador, Costa Rica, Guatemala, Nicarágua, Peru e Bolívia foram, durante anos, os países pioneiros nessa discussão com relação à formulação de instrumentos legais e de políticas públicas específicas para o TBC. Todavia, os avanços no sentido de se estabelecer um marco legal para o TBC nos contextos nacionais só foram possíveis devido à ação articulada por meio de redes, federações e associações locais, regionais e nacionais, conforme se buscará discutir a seguir neste artigo.

\section{A experiência da Rede de Turismo Comunitário da América Latina (REDTURS)}

O reconhecimento da importância das redes ganhou força e vem adquirindo novos significados, principalmente, a partir do século $X X$, no contexto dos debates decorrentes da revolução da tecnologia, da informação e do conhecimento na sociedade contemporânea.

Diversas perspectivas teóricas sobre a noção de rede (LOMNITZ, 2009; CASTELLS, 2010; SCHERER-WARREN, 2007) possibilitam entendêla como uma nova estrutura social e de poder, que é também resultado da expressão e da organização de movimentos sociais, ações coletivas e organizações da sociedade civil. A partir dessa referência, é possível considerar que o entendimento sobre redes implica a análise de relações e de alianças formais e informais, baseadas, muitas vezes, em princípios de solidariedade e confiança para a promoção de intercâmbio e compartilhamento de informação, conhecimento, capitais e recursos no processo de organização coletiva de grupos sociais em diversos contextos, muitos deles de desigualdade e vulnerabilidade social, como ocorrem em 
muitos casos de iniciativas de turismo de base comunitária na América Latina.

O reconhecimento da importância da institucionalização e da promoção de redes de turismo comunitário, nas esferas nacionais e locais, pelos países latino-americanos, foi expresso na Declaración de Otavalo sobre Turismo Comunitario, sustentável, competitivo e com identidade cultural, documento assinado por lideranças de comunidades tradicionais da Bolívia, Equador e Peru, durante o I Encuentro Técnico Internacional sobre Gestión del turismo sostenible y competitivo: Alianzas entre Estado, Empresa y Comunidad, realizado no Equador, em 2001, pela REDTURS. Essa pode ser considerada a primeira iniciativa de articulação em rede para a promoção dos destinos turísticos comunitários na América Latina.

Em sua origem, a REDTURS foi criada pela OIT em resposta às aspirações de diversas organizações indígenas e rurais, cujo propósito foi intervir sobre a prática do TBC e promover assistência técnica e respaldo institucional para facilitar o acesso de pequenos negócios rurais a serviços de desenvolvimento empresarial e a novos mercados. Com esse objetivo, a REDTURS tem como missão apoiar os processos de formação e de fortalecimento de redes de turismo comunitário em países da América Latina para diversificar as possibilidades de emprego e renda, valorizar a cultura local e promover o associativismo. Esta representa uma rede aberta formada por comunidades rurais e indígenas, instituições de apoio e profissionais do setor que compartilham uma leitura similar de TBC, através da qual se busca compatibilizar a eficiência econômica com os princípios de equidade social, de valorização das identidades culturais e de preservação dos recursos naturais (MALDONADO, 2007).

Com base nessa perspectiva, foram organizados, posteriormente à sua fundação, quatro encontros consultivos regionais (Costa Rica, 2003; Panamá, 2005; Bolívia, 2007; Guatemala, 2008), com o apoio governamental e a participação de mais de 20 organizações de 13 países latino-americanos, nos quais ocorreram importantes discussões sobre a realidade do TBC para a região. Nesses encontros, foram criadas regras para incrementar processos associativos que procuram uma inserção competitiva da oferta de serviços nos mercados e fomentam o uso sustentável do patrimônio natural e cultural, priorizando também, por meio da qualificação dos recursos humanos e da formação de lideranças comunitárias, a busca pela melhoria da capacidade de autogestão (MALDONADO, 2007).

Apesar de alguns avanços desde então, Maldonado (2007) ressalta uma série de dificuldades para o desenvolvimento do TBC em rede na América Latina. Essas são derivadas da limitada capacidade de gestão das redes de turismo comunitário, da falta de qualidade da oferta de prestação de serviços com relação à demanda, da elevada dependência de recursos externos, da limitação de membros envolvidos no processo, do estabelecimento de relações informais, da inexistência de políticas públicas específicas para esse tipo de iniciativa e da forte influência do setor turístico convencional, baseado na lógica do mercado nesse contexto. 
Para enfrentar esses problemas, no $V$ Encuentro consultivo regional da REDTURS, ocorrido em 2007, na Bolívia, reconheceu-se que as redes locais e nacionais devem ser as próprias gestoras das alianças e dos intercâmbios realizados. Para tal, foi questionada a validade da formalização dessas redes para que sejam as próprias interlocutoras oficiais do turismo comunitário no seu país, sejam elas denominadas como rede propriamente dita ou como câmara, federação, apenas para citar algumas (OIT, 2008), conforme estão dispostas na lista a seguir:

1.RITA: Red Indígena de Turismo de México.

2.SENDA SUR: Red de Turismo de Chiapas Ecotours y Etnias, México.

3.FENATUCGUA: Federación Nacional de Turismo Comunitario de Guatemala.

4.MUTU: Red de Turismo Comunitario Garífuna, Honduras.

5.RENITURAL: Red Nicaragüense de Turismo Rural.

6.ACTUAR: Asociación Costarricense de Turismo Rural Comunitario.

7.COOPRENA: Red de Turismo Rural Comunitario de Costa Rica.

8. Red de Turismo de los Pueblos KUNA, Panamá.

9.KAÍ ECOTRAVEL: Operador Turístico Comunitario, Colombia.

10.FEPTCE: Federación Plurinacional de Turismo Comunitario de Ecuador.

11. Asociación de Turismo Comunitario Llachón, Puno, Perú.

12.TUSOCO: Red Boliviana de Turismo Solidario Comunitario.

13.TURISOL: Red Brasileña de Turismo Solidario Comunitario.

14. Red de Parques Comunitarios Mapu Lahual, Chile.

15.Red de Turismo Campesino de los Valles Calchaquíes, Salta, Argentina.

16. Red Provincial de Turismo Rural de Base Comunitaria de Jujuy, Argentina.

Fonte: Maldonado (2007, p. 11).

A REDTURS desenvolveu algumas iniciativas para que os governos nacionais, em estreita colaboração com as organizações comunitárias, reconhecessem e difundissem os códigos de conduta em turismo, acordados e deliberados em várias edições de seus encontros consultivos e reuniões técnicas. Sendo assim, a REDTURS buscou desenvolver suas ações orientadas pelo seu Plano de Ação 2003-2010, que definiu cinco eixos estratégicos: 1) Garantia do reconhecimento dos direitos das comunidades indígenas e rurais nas políticas e programas de turismo; 2) Garantia dos 
benefícios econômicos derivados do turismo às populações locais envolvidas; 3) Preservação ambiental e integridade dos territórios das populações indígenas e campesinas; 4) Preservação da autenticidade e valorização da riqueza do patrimônio cultural local; 5) Fortalecimento das instituições, das formas de participação e da coesão social comunitária mediante o turismo.

Considerando esses caminhos possíveis em planejamento, a construção coletiva de redes de TBC vem sendo desenvolvida como uma estratégia de resistência ao turismo de massa, que ameaça o equilíbrio sociocultural e ambiental, e à pressão internacional pela implementação de projetos de complexos hoteleiros e da especulação imobiliária. Além disso, parece que essas redes se traduzem como uma alternativa para minimizar as práticas de agricultura intensiva e para conter a migração de jovens do campo para as cidades. Isso implica, ainda, a capacidade de essas redes influenciarem políticas públicas para o fortalecimento do turismo comunitário. Também em busca de formas autônomas de comercialização do TBC, algumas redes têm criado a sua própria operadora e agência de turismo, investindo nos principais mercados turísticos emissores Estados Unidos e Canadá, bem como no público nacional interessado (OIT, 2008).

Esse processo, contudo, tem estado sujeito a um forte processo de desmobilização, principalmente a partir de 2011, quando as ações de coordenação da REDTURS pela OIT foram encerradas, colocando em evidência a sua condição de informalidade, uma fragilidade para a sua consolidação. Entretanto, essa situação também vem provocando que as redes nacionais e locais pesquisadas se articulassem e se apoiassem entre si mesmas, considerando que é importante contar com uma "rede de redes", ou seja, uma rede latino-americana que congregue redes nacionais e regionais (REDTURS, 2014).

Para reforçar esse movimento em rede do TBC na América Latina, em 2014, foi promovido o Fórum Internacional de Turismo Solidário e Desenvolvimento Sustentável - FITS² América Latina 2014, na Nicarágua. Na Declaración de Granada, resultante desse encontro, foi afirmada que a reativação da coordenação executiva da REDTURS, por meio da definição de uma pessoa jurídica para formalizar o processo e o papel dos seus membros, seja uma estratégia fundamental para o fortalecimento das redes locais e nacionais (FITS, 2014).

Diante dos resultados alcançados nesse Fórum, foram assumidos compromissos para o avanço do turismo comunitário em redes, no que tange ao intercâmbio de ideias e de informações, ao fortalecimento das atividades locais, à gestão de fundos e às "boas" práticas ambientais e sociais, que têm sido apoiados por iniciativas da gestão pública, de ONGs, de fundações, de empresas e de agências internacionais (FITS América Latina, 2014).

Nesse sentido, reconheceram-se as potencialidades para a implementação futura de redes de TBC, sobretudo com relação ao aproveitamento do contexto de proteção da natureza e cultura em que estão inseridos os empreendimentos turísticos; ao uso dos princípios de economia 
social e solidária; à facilidade da entrada de estrangeiros em alguns países; à valorização dos recursos naturais e da inclusão social e ao interesse por parte dos turistas em produtos artesanais e na gastronomia local (FITS América Latina, 2014).

Por outro lado, reforçaram alguns fatores limitantes à formação de redes em turismo comunitário. São eles: deficiência de políticas públicas e de incentivos pelo governo para o avanço das redes de turismo; presença de conflitos entre a comunidade no processo de tomada de decisão; ausência de projetos que envolvam a comunidade local; baixo nível de escolaridade dos comunitários envolvidos; carência de infraestrutura básica; escassez de serviços de telecomunicação em determinados locais; dificuldades para intercâmbios com base em encontros e conhecimentos que poderiam ser articulados entre os países, entre outros (FITS América Latina, 2014).

De todo modo, pela presente pesquisa, entendeu-se a possibilidade de se estabelecer uma estratégia comum, respeitando as particularidades de cada iniciativa. Isso pode implicar o mapeamento das experiências e a identificação do que deu certo, no sentido de ser ter um direcionamento político para o desenvolvimento de redes de TBC na América Latina.

\section{Considerações finais}

No presente artigo, refletiu-se sobre as configurações do movimento de turismo de base comunitária (TBC) em rede na América Latina, com ênfase no caso da REDTURS. Nesse esforço de pesquisa, vale ressaltar que informações detalhadas e pesquisas sobre a gênese e o funcionamento de redes de turismo comunitário na América Latina são ainda limitadas ou quase inexistentes, o que reforça a importância de pesquisas acadêmicas com esse objetivo. Além disso, são também de difícil acesso os dados estatísticos sobre os empreendimentos de TBC organizados em rede e o acompanhamento de seus impactos econômicos, sociais, ambientais e culturais.

A REDTURS pode ser considerada a primeira iniciativa de articulação em rede para a promoção dos destinos turísticos comunitários na América Latina. Considerando o contexto da REDTURS, da origem ao momento atual, concluiu-se que os nós das iniciativas de redes de TBC em curso estão sempre por se fazer, sendo articulados e reforçados pelos atores sociais envolvidos. Apesar disso, observaram-se inúmeras fragilidades e potencialidades para o fortalecimento do movimento em rede de TBC.

Pela presente pesquisa, a REDTURS passa por uma fase inativa, mediante o encerramento, em 2011, das ações de coordenação promovidas pela OIT, o que dificultou a concretização das ações planejadas. Essa situação remete a questionar em que medida essas iniciativas externas têm efetivamente fomentado o protagonismo dos atores sociais locais envolvidos na construção das redes de TBC. Além disso, essa reflexão requer a compreensão dos reais interesses das agências internacionais, como a OIT, para com iniciativas desse tipo, bem como sinaliza outra lacuna referente à forma de enfrentar essa questão de institucionalização de uma rede, tendo em vista, por premissa, o seu caráter dinâmico de construção coletiva. 
A despeito das fragilidades, ainda assim, reconheceu-se que a REDTURS foi fundamental para congregar várias redes nacionais e locais para a discussão sobre documentos norteadores, para o intercâmbio de experiências na prática turística e para o estabelecimento de um marco legal para a prática do TBC em seus respectivos contextos de inserção. Isso porque o fortalecimento dessas iniciativas de redes implica a existência de políticas públicas e de marcos normativos adequados para 0 desenvolvimento dessas redes na América Latina.

De todo modo, o movimento em rede de TBC na América Latina, como a REDTURS, tem envolvido comunidades rurais e indígenas, instituições de apoio e profissionais do setor, traduzindo-se, nesta reflexão, como uma possível estratégia de poder e resistência, tendo como bandeira comum o turismo de base comunitária.

\section{Referências bibliográficas}

CASTELSS, M. A sociedade em rede. São Paulo: Paz e Terra, v. 1, 6 a $^{\mathrm{a}}$ edição, 2010.

FITS. Foro Internacional de Turismo Solidario y Desarrollo Sostenible América Latina. Declaración de Granada. Granada, Nicarágua, 25 de septiembre del 2014. Disponível em http://fitstourismesolidaire.org/2014/nica2014.asp. Acesso em: 15 de julho de 2016.

FITS América latina. Taller Internacional FITS Balance y propuestas. Granada, Nicaragua. 14 de septiembre de 2014. 2014. Disponível em http://fits-tourismesolidaire.org/2014/nica2014.asp. Acesso em: 15 de julho de 2016.

LIMA, L.C.; CORIOLANO, L.N.M.T. (Orgs.) Turismo e Desenvolvimento Social Sustentável. Fortaleza: EDUECE, 2003.

LOMNITZ, L.A. Redes sociais, cultura e poder. Rio de Janeiro: E-papers, 2009.

MALDONADO, C. Workshop planejamento e gestão de empreendimentos turísticos de base comunitária REDTURS. In: INOVABR, Inovação Social e Sustentabilidade. Rio de Janeiro: COPPE/UFRJ, 22-26, nov. 2010.

MALDONADO, C. O turismo rural comunitário na América Latina: Gênesis, características e políticas. In: BARTHOLO, R.; SANSOLO, D.; BURSZTYN, I. (Orgs). Turismo de base comunitária: diversidade de olhares e experiências brasileiras. Rio de Janeiro: Letra e Imagem, 2009, p. 25-44.

MALDONADO, C. Fortalecendo as Redes de Turismo Comunitário REDTURS - na América Latina. Turismo Sustentável e Desenvolvimento Local. Turim, Itália: Centro Internacional de Formação da OIT, @local.glob, 4, 08-14, 2007.

MENDONÇA, T.C.M.; IRVING, M.A. Turismo de base comunitária: a participação como prática no desenvolvimento de projetos turístico no Brasil - Prainha do Canto Verde, Beberibe (CE). Caderno Virtual de Turismo, vol. 4 (4), 12-22, 2004. 
MENDONÇA, T.C.M.; MORAES, E.A. Reflexões emergentes sobre turismo de base comunitária, à luz da experiência no "Paraíso Proibido": Vila do Aventureiro, Ilha Grande, Brasil. Journal of Tourism and Development 4, 2012, 1169-1183.

NEUHAUS, E.; SILVA, J.S. (org). Um outro turismo é possível! Reflexões sobre desigualdades, resistências e alternativas no desenvolvimento turístico. Fórum Social Mundial Porto Alegre 2005. Fortaleza: FBOMS/Instituto Terramar, 2006.

ORGANIZACIÓN INTERNACIONAL DEL TRABAJO. Gobiernos locales, turismo comunitario y sus redes. Memória $\mathrm{V}$ Encuentro consultivo regional (REDTURS). Ginebra: OIT, Documento de Trabajo núm. 12, 2008.

ORGANIZAÇÃO INTERNACIONAL DO TRABALHO. Convenção sobre Povos Indígenas e Tribais (No. 169). Geneva: OIT, 1989.

OMT. Carta do Turismo Sustentável. Conferência Mundial de Turismo Sustentável. Lanzarote, Ilhas Canárias, Espanha, de 27 a 28 de abril de 1995.

REDTURS. Declaración de San José sobre Turismo Rural Comunitario. San José de Costa Rica, el 28 de octubre 2003. Disponível em: http://www.redturs.org/inicio/docu/DeclaracionSan-Jose-Espa.pdf. Acesso em: 22 de abril de 2016.

REDTURS. Declaración de Otavalo sobre Turismo Comunitario, sustentável, competitivo e com identidade cultural. Otavalo, Ecuador, 14 de septiembre del 2001. Disponível em: http://www.redturs.org/documentos/Otavalo.pdf. Acesso em: 22 de abril de 2016.

REDTURS. Acta de Reunión REDTURS. Granada, 24 de septiembre de 2014.

SCHERER-WARREN, I. Redes sociais e de movimentos. In: FERRARO Jr., Luiz Antonio. (Org). Encontros e Caminhos: Formação de Educadoras(es) Ambientais e Coletivos Educadores. Brasília: Ministério do Meio Ambiente, Departamento de Educação Ambiental, vol. 2, 2007, pp. 325-332.

UNWTO. World Tourism Organization. Tourism and Poverty Alleviation. ST-EP Programme, vol. 1, Madrid, Spain: UNWTO, 2002. Disponível em: http://www.e-unwto.org/doi/pdf/10.18111/9789284405497. Acesso em: 17 de setembro de 2016.

\section{Notas:}

${ }^{1}$ Importante ressaltar que outros documentos norteadores para uma reflexão ampla sobre o turismo de base comunitária na América Latina se referem à Carta para um Turismo Sustentável de Lanzarote (OMT, 1995) e ao Programa de Turismo Sustentável e Eliminação da Pobreza (ST-EP) (UNWTO, 2002).

2 Os Fóruns Internacionais de Turismo Solidário e Desenvolvimento Sustentável (FITS) são iniciativas, no plano global, em apoio às formas 
alternativas de desenvolvimento turístico, que vêm se sucedendo desde 2003 (em Marselha/França), envolvendo a difusão de iniciativas públicas e privadas e empreendimentos comunitários de várias regiões do mundo, com o objetivo de ampliar a discussão sobre o turismo solidário e sustentável e de firmar alianças entre os diversos setores da sociedade para influenciar políticas públicas intersetoriais, adequadas às diferentes realidades nacionais.

Edilaine Albertino Moraes: Universidade Federal de Juiz de Fora, Juiz de Fora, MG, Brasil.

E-Mail: edilaineturmoraes@hotmail.com

Link para o currículo Lattes: http://lattes.cnpq.br/5450933897214464

Marta de Azevedo Irving: Universidade Federal do Rio de Janeiro, Rio de Janeiro, RJ, Brasil.

E-Mail: marta.irving@mls.com.br

Link para o currículo Lattes: http://lattes.cnpq.br/1912229324377473

Joana da Silva Castro Santos: Universidade Federal do Rio de Janeiro, Rio de Janeiro, RJ, Brasil.

E-Mail: joanascsantos@gmail.com

Link para o currículo Lattes: http://lattes.cnpq.br/2799821399042072

Hugo Quintanilha Silva Santos: Universidade Federal do Rio de Janeiro, Rio de Janeiro, RJ, Brasil.

E-Mail: hugoqts@gmail.com

Link para o currículo Lattes: http://lattes.cnpq.br/6141779875302059

Maycon Correia Pinto: Universidade Federal do Rio de Janeiro, Rio de Janeiro, RJ, Brasil.

E-Mail: mayconcorreia2@gmail.com

Link para o currículo Lattes: http://lattes.cnpq.br/8667449690841046

Data de submissão: 30 de agosto de 2016

Data de recebimento de correções: 27 de novembro de 2016

Data do aceite: 27 de novembro de 2016

Avaliado anonimamente 